\title{
A Wirelessly Powered Micro-Spectrometer for Neural Probe-Pin Device
}

\author{
Sang H. Choi \\ NASA Langley Research Center, Hampton, VA, USA \\ Min Hyuck Kim, Kyo D. Song, Hargsoon Yoon \\ Department of Engineering, Norfolk State University, 700 Park Avenue, Norfolk, VA, USA \\ Uhn Lee \\ Integrated Brain Research Institute, Gachon University, Incheon, South Korea
}

\begin{abstract}
Treatment of neurological anomalies, places stringent demands on device functionality and size. A micro-spectrometer has been developed for use as an implantable neural probe to monitor neuro-chemistry in synapses. The microspectrometer, based on a NASA-invented miniature Fresnel grating, is capable of differentiating the emission spectra from various brain tissues. The micro-spectrometer meets the size requirements, and is able to probe the neuro-chemistry and suppression voltage typically associated with a neural anomaly. This neural probe-pin device (PPD) is equipped with wireless power technology (WPT) enabling operation in a continuous manner without requiring an implanted battery. The implanted neural PPD, together with a neural electronics interface and WPT, allow real-time measurement and control/feedback for remediation of neural anomalies. The design and performance of the combined PPD/WPT device for monitoring dopamine in a rat brain will be presented to demonstrate the current level of development. Future work on this device will involve the addition of an embedded expert system capable of performing semi-autonomous management of neural functions through a routine of sensing, processing, and control.
\end{abstract}

Keywords: Optical neural sensing, microspectrometer, neurotransmitter, wireless power transfer

\section{INTRODUCTION}

A clear understanding of the neurodegeneration mechanisms in a dopaminergic neural system is essential for the development of novel therapeutic treatment strategies to prevent irreversible loss of neuronal functions. According to previous investigations with electrophysiological, pathological, radiological and virological studies, neurodegeneration including cognitive and motor function decline, depression and memory loss is highly associated with the loss of neuronal function of basal ganglia (BG) $[1,2]$. The BG is a group of subcortical neural structures including the striatum, pallidum, substantia nigra, and subthalamic nucleus that help control motor and cognitive functions. Even though several other areas of the brain are also involved in the complex abnormalities, the neuron death and morphological alterations in the BG, especially dopaminergic neuronal system, have been linked to depression and Parkinsonian motor function decline. To counteract the loss of dopamine (DA) levels caused by dopaminergic neuronal cell loss, symptomatic treatments modulating the dopamine reuptake process and dopaminergic neurotransmission are available. However, side effects, such as addiction and dyskinesias are a concern, and even neurotoxicity has been reported including aberrant immune response and oxidative stress [3].

Recent technology developments are offering great potentials to treat neurological disorders and diseases. One of these important technology developments, Deep Brain Stimulation (DBS), demonstrated a new way to treat neurological disorders, especially for Parkinson's Disease (PD). Around 30,000 DBS, operations have been carried out world-wide with great success rates. DBS uses implantation of electrodes precisely at the neuro-anatomical targets of this disease, and a pulse of current through these electrodes instantly mitigates or stops the tremors and releases the frozen muscles which are characteristic of the disease [4]. However, current DBS technology shows several limitations such as lack of automatic 
parameter adjustment and the inability to continuously adapt to the clinical fluctuations which is a typical phenomenon of advanced Parkinson's disease. Therefore, adaptive DBS should be able to deliver certain functions interactively when they are needed, and at the same time respond automatically when a patient's symptoms are varied.

Currently, there are no sensory feedback systems available for DBS applications. To meet this need, a company, Medtronic Inc., Minnesota, announced the first implantable DBS system that enables the sensing and recording of select brain activity to provide targeted DBS therapy. However, electrical sensing of local field potentials can suffer from the residual effect of suppression voltage provided for the electrical stimulation. The large DBS pulses can generate artifacts in the measurements. In addition, sensor lifetime can be an issue. Implanted electrodes are vulnerable to electrode fouling, corrosion, or sensing signal variations caused by localized chemical environment changes from the foreign body response after implantation. Therefore, chronic recording capable neurotransmitters receive a great deal of attention for advanced DBS research that will offer better clinical treatments for neurological disorders.

This paper presents a wireless powered micro-spectrometer for neural probe pin devices (n-PPD) which can monitor the microscopic alteration of dopaminergic neuronal function. This research is particularly focused on real time monitoring of dopamine in the brain for a long period of time without having to change the battery after implantation in the body. This optical neural sensing system, coupled with wireless power transfer technology was designed for future integration with a neuro-stimulation unit for clinical applications, as shown in a diagram in Figure 1. The wireless power transmission technology considered for the n-PPD is based on the principles of rectenna [5] and magnetic resonance coupling [6], which will eventually alleviate the use of implanted batteries requiring periodic replacement. Wireless power transmission (WPT) using an electromagnetic wave was originally demonstrated by Tesla [7]. Tesla used a coil that was connected to a $60 \mathrm{~m}$ tower, fed by a $1300 \mathrm{~kW}, 150 \mathrm{kHz}$ power source. Later, rectenna-based WPT technology using rectenna was developed in the 1960s by Brown [5], which can focus high frequency electromagnetic waves and effectively deliver toward the power receiver.

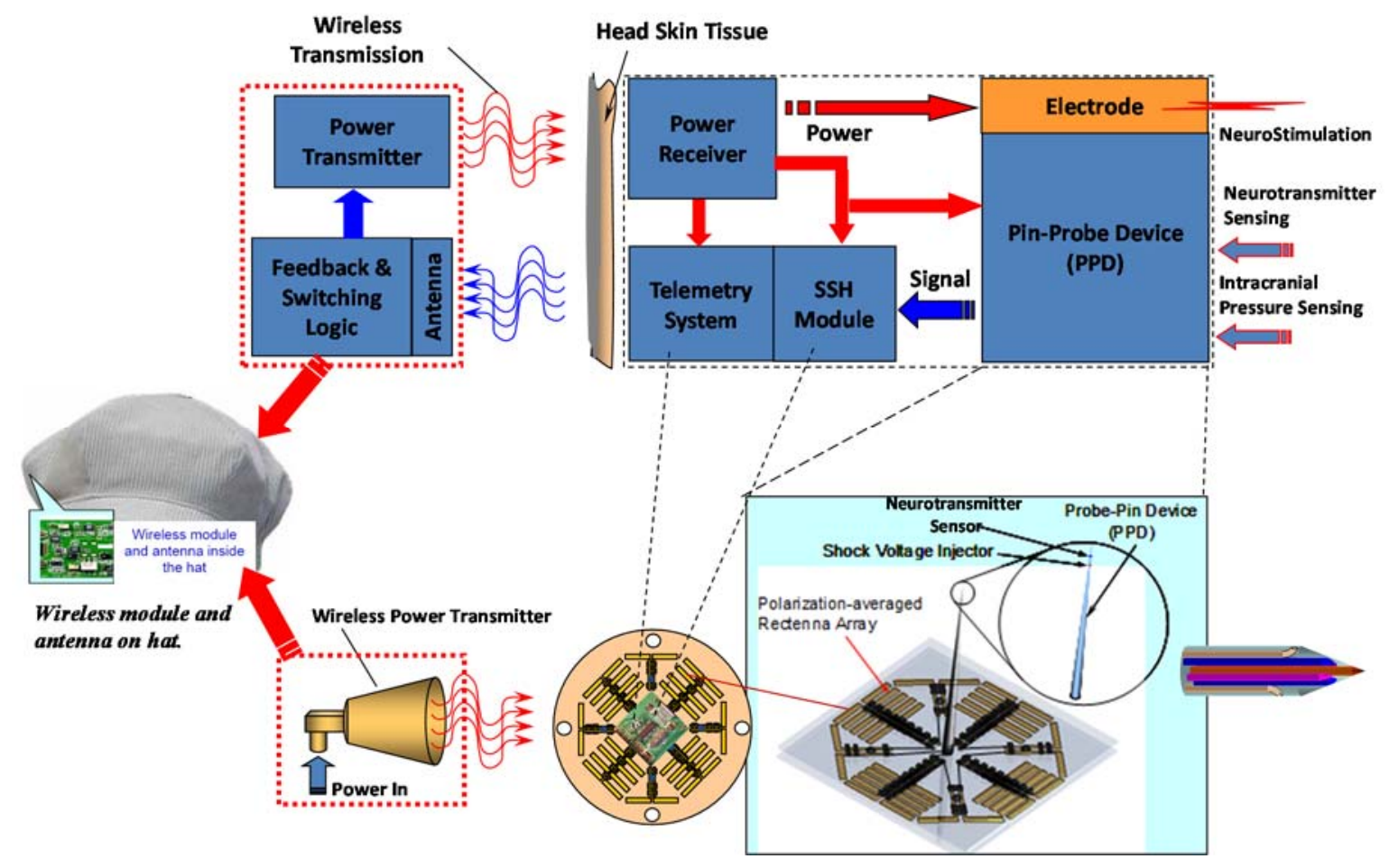

Figure 1. Conceptual diagram of an adaptive neural sensing and stimulation system. 


\section{MICROSPECTROMETER AND PROBE PIN DEVICE}

A small spectrometer with a prism or grating has been applied for various applications from analysis of materials to biomedical research [8]. Cubic inch mini-spectrometers are commercially available from Hamamatsu Photonics with 9 $\mathrm{nm}$ spectral resolution. Various compact spectrometers have also been developed by many other research groups such as Chaganti et al. [9], Montgomery et al. [10], and Kitaura et al. [11]. Although most of today's spectrometers use the Fraunhofer diffraction principle, we utilized the fundamental advantage of Fresnel diffraction for miniaturization of spectrometers [12-14]. Additionally, we developed an electronic data acquisition unit with geometric optic modules for real time optical sensing of the brain through wireless communication. Here, a bluetooth wireless communication module, and a power management unit were integrated with a PSOC-5 microcontroller [14]. An image of the optical dopamine sensing system is shown in Figure 2(a).

For optical sensing of neurotransmitters, an n-PPD is constructed with a micro-spectrometer as a core component that utilizes the Fresnel diffraction principle. Here, the n-PPD device performs the spectroscopic detection of fluorescence from the dopamine molecule in order to measure and discern the variation in dopaminergic transmittal synapse. To realize fluorescent sensing capability in the brain, the sensing system is designed to be small and implanted on the skull surface. For high sensitivity and sensing selectivity, a sensor constructed using fluorescent nano-structures and materials (nanostructures of quantum-dot or plasmonic patch arrays) were integrated at the tip of optical fiber to capture and measure the signal locally, rather than guiding the fluorescence signal through the optical fiber. With the functionality of nanomaterials, observation of physiological changes in dopamine concentration is possible at the micro/nano-mole scale. The micron-scale diameter optical fiber, used in many biomedical sensing applications, were physiologically adaptive to biological systems making for an ideal interface with neural cells to effectively transfer the optical signals from the light source to sensor array. A pulse mode of blue LED light with the wavelength of $475 \mathrm{~nm}$ is sent into the brain through an optical fiber to stimulate and emit fluorescence from dopamine. An optical probe and a filter for the emission spectra measurement was set for the transmission of 500-700 nm wavelength fluorescence signals in Figure 2(a).

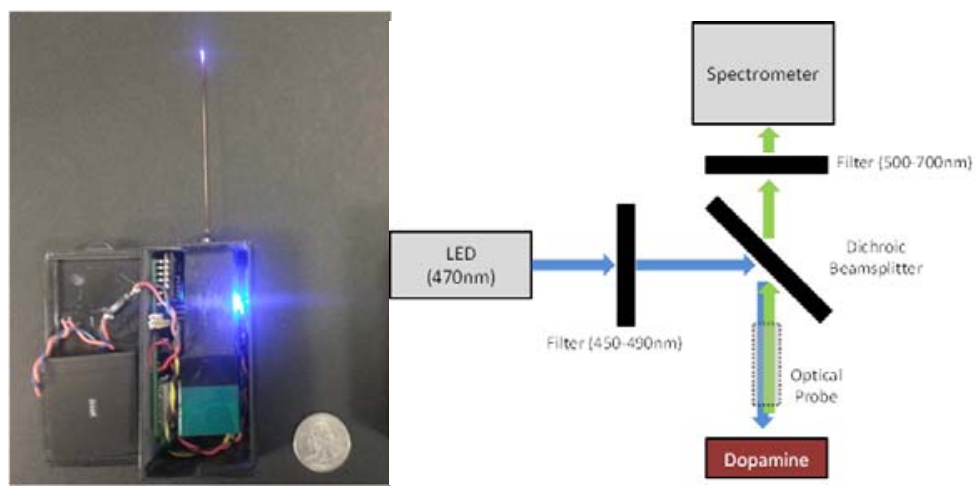

(a)

(b)

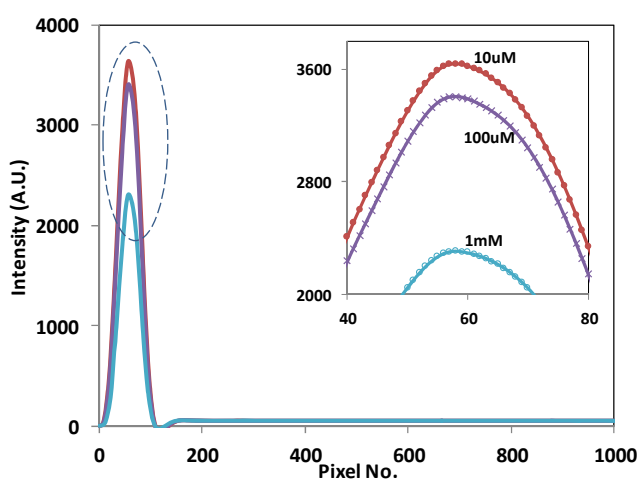

(c)

Figure 2. (a) An image of optical dopamine sensing system, (b) sensor diagram, (c) dopamine sensing results.

The small and narrow optical fiber probe can reach to any region of brain. Furthermore, optical fibers can be built into a sensor array form. Many different types of optical fibers, such as flat, angled and tapered fibers, have been implemented for various sensing targets at the same time. By functionalizing the tip surface using nanoscale fluorescent materials, an $\mathrm{n}$-PPD and its associated system could detect micromole concentrations of dopamine as shown in Figure 2(c). 


\section{WIRELESS POWER TRANSMISSION (WPT) AND MANAGEMENT}

In this research, we have developed two different WPT devices, rectenna and magnetic resonance coupling, for remote and near field wireless power transfer.

\section{3-1. Power Management Circuit}

In addition, for optimum wireless power transfer and power management, a feedback controlled algorithm was applied in the power management system in Figure 3. Depending on the distance and physical angle of the transceivers, it is desirable to control the source power level and frequency, even the direction of radiation. When the designed power output conditions are met, the control system needs to select modes for system operation or charging a power storage device. For the proof of concept, we developed an algorithm for the embedded microcontroller PSoC-5 to measure the output voltage after rectification of electromagnetic waves and adjust the frequency of the source waveform generator.

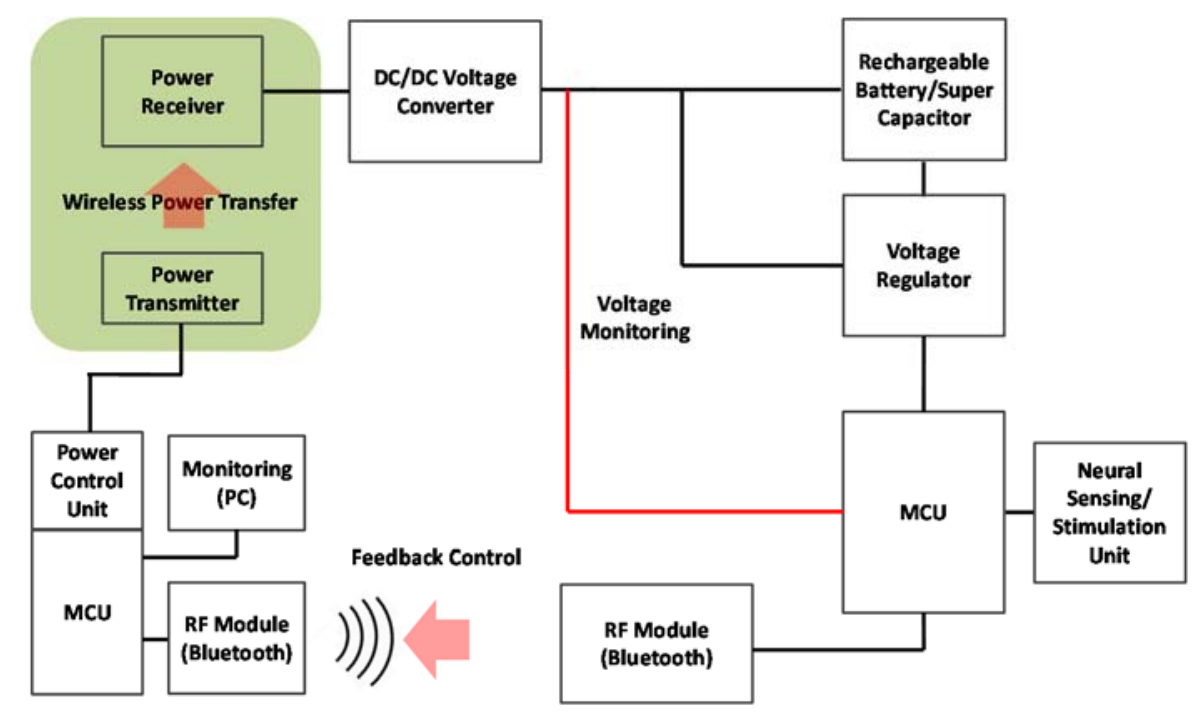

Figure 3. Schematic diagram of a feedback controlled wireless power transfer system.

\section{3-2. WPT using Rectenna Array}

A rectifying antenna, called a rectenna, consists of an antenna part and a rectifier consisting of a Schottky barrier diode and a capacitor as shown in Figure 4. A dipole rectenna uses two metallic conductors that carry the charge coupled with an electromagnetic wave. The power output of a rectenna is determined by the Schottky barrier diode of the rectifying circuit and the antenna efficiency. For our device, we connected a dipole rectenna array for WPT. The half-wave of the

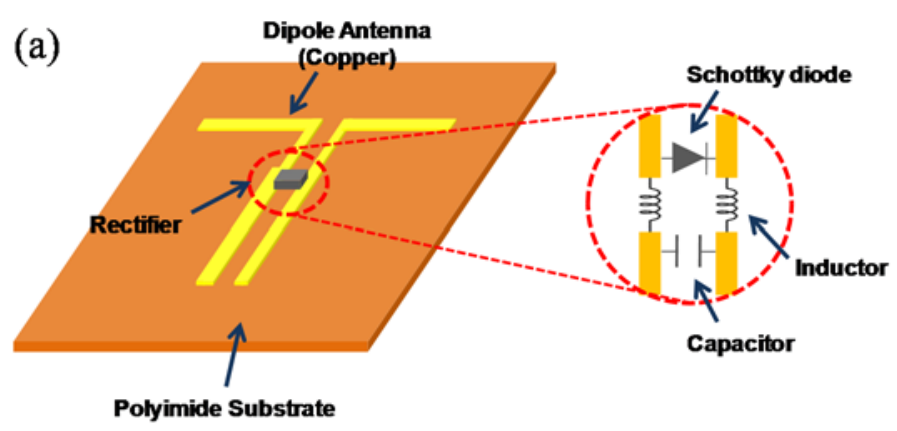

(b)

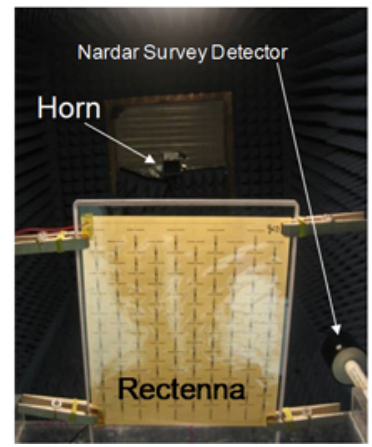

Figure 4. (a) Schematic diagram of a rectenna unit and (b) rectenna array tested. 
dipole antenna was designed for $10 \mathrm{GHz}$ frequency operation. The forward current of the Schottky diode (MA4E2054$1141 \mathrm{~T}, \mathrm{M} / \mathrm{A}-\mathrm{COM}$ ) used for this rectenna at room temperature was selected to have $20 \mathrm{~mA}$ output.

The rectenna circuits were patterned on a flexible polyimide foil, $20 \mu \mathrm{m}$ thick with an $18 \mu \mathrm{m}$ thick copper layer, using lithography followed by chemical etching. Schottky diodes were soldered to the dipole rectenna pattern. The size of the rectenna patch was $18 \mathrm{~cm} \times 19 \mathrm{~cm}$, and the weight of a single patch was $3.0 \mathrm{~g}$. Fabricated rectenna arrays were tested individually in an anechoic chamber. A combination of signal generator (HP-8672A) and amplifier (VZX-6983-G5GLM, TWT, Varian) injected $200 \mathrm{~W}$ of microwave power at a frequency range of $7-18 \mathrm{GHz}$ to irradiate the rectenna array through a Narda rectangular horn antenna. The output voltage and current were measured at various frequencies, distances and polarization conditions. According to the evaluation of 16 different rectennas, the average output power for a rectenna patch was $360 \mathrm{~mW}(3.0 \mathrm{~V}, 185 \mathrm{~mA})$ at $10.3 \mathrm{GHz}$ satisfying the power requirement of the optical sensing system.

The rectenna array was tested by placing it behind a pig skin to determine the transmission of microwave power through living tissue for implanted devices. Figure 5 shows the test result of the rectenna array that was placed behind a pig skin. The test was to determine the attenuation rate of microwave power through a pig skin. The results show that the attenuation through a pig skin at $9.3 \mathrm{GHz}$ is substantially lower and $0.03 \mathrm{~mW}$ of power is passed through the skin after receiving 2 $\mathrm{mW}$ incident wave (left of Figure 5). This is a very encouraging sign for wireless power transmission that can be applicable to the implanted medical devices. If a couple of wattage power is used as a beam power source, $30 \mathrm{~mW}$ of power can be harvested at a rectenna array. This level of power harvest from an implanted rectenna array is sufficient to operate an implanted medical device, or to store the energy into batteries of devices. This type of wireless power transmission technology based on a rectenna array is also ideal for far-distance applications.
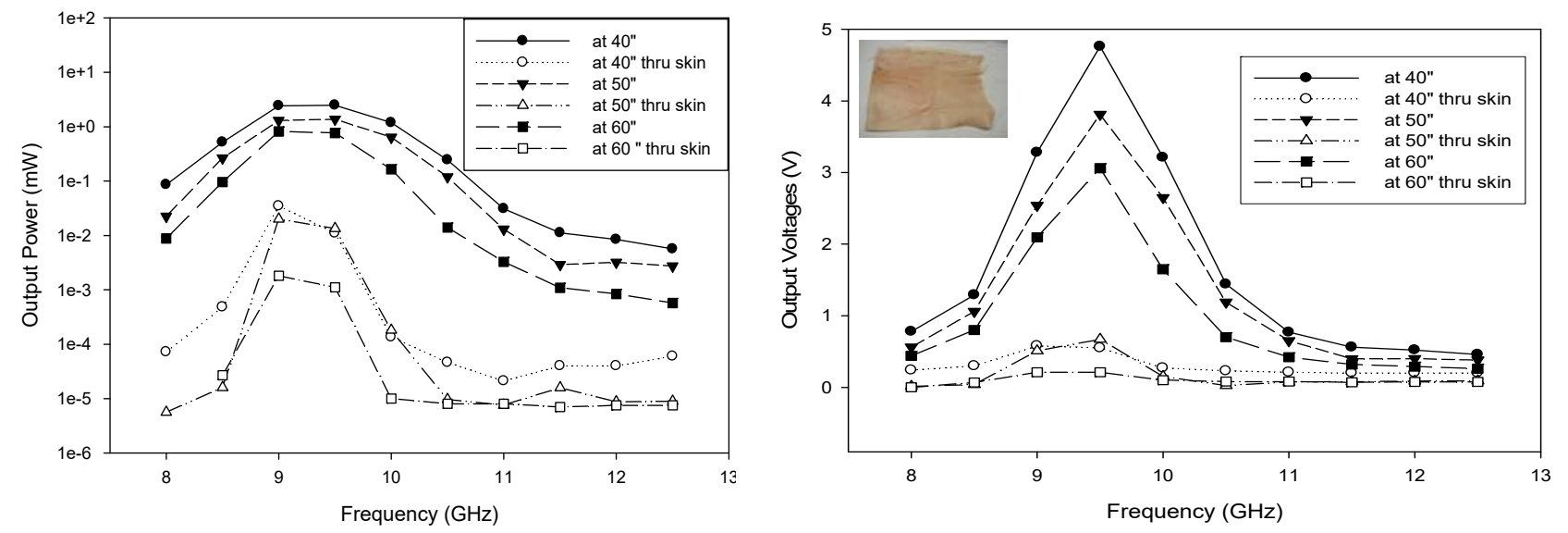

Figure 5. Test results of rectenna array power reception behind pig skin. (Left) The power received by rectenna array through pig skin measured along the distance from the injection horn. (Right) Voltage output from rectenna array after attenuating through pig skin.

\section{3-3. WPT using Magnetic Resonance Coupling}

In recent years, a significant improvement in WPT by magnetic resonance coupling was demonstrated with over $50 \%$ of power delivery efficiency in a proximity power delivery. Compared to conventional inductive coupling, magnetic resonance coupling uses the resonance effect on the primary and secondary coils. In this research, various magnetic resonance coupling WPT devices were investigated. In device design, magnetic resonance coupling effects can be calculated by the Coupled Mode Theory [6]:

$$
\dot{a}_{m}(t)=\left(i \omega_{m}-\Gamma_{m}\right) a_{m}(t)+\sum_{n \neq m} i \kappa_{m n} a_{n}(t)+F_{m}(t)
$$

where $m$ and $\mathrm{n}$ are the resonant coils, $\omega_{m}$ the resonance frequency, $\Gamma_{m}$ intrinsic decay rate at the object, and $\kappa_{m n}$ coupling coefficients between the resonant objects. The intrinsic decay $\Gamma_{m}$ is given by the absorption and radiated losses in the 
system. In this research, we limited our experiment to the case of two resonant objects. When the source and receiving coils are resonant, the power transfer efficiency $\eta$ is

$$
\eta=\frac{\Gamma_{W}\left|a_{D}\right|}{\Gamma_{S}\left|a_{S}\right|^{2}+\left(\Gamma_{D}+\Gamma_{W}\right)\left|a_{D}\right|}
$$

where $\Gamma_{\mathrm{W}}$ is the decay rate due to the load resistor, $\Gamma_{\mathrm{S}}$ and $\Gamma_{\mathrm{D}}$ are the intrinsic decay rates of the source and receiving coils, respectively, and, $a_{S}$ and $a_{D}$ are the field amplitude of the source coil and the receiving coil. A schematic diagram of the system is shown in Figure 6.

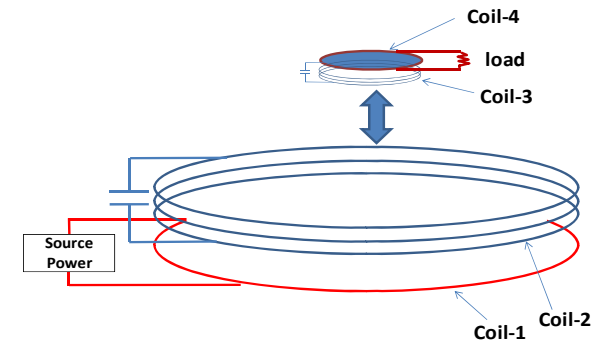

[1]

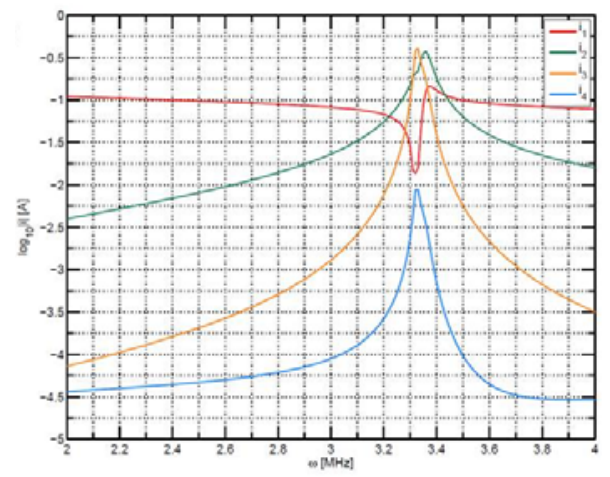

(c)

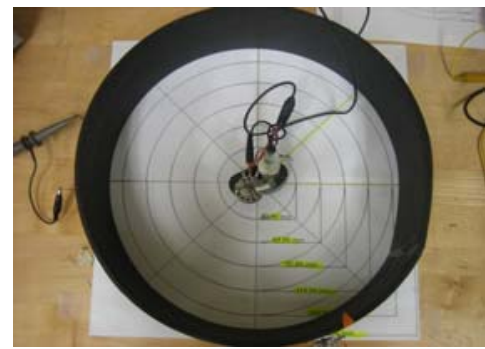

(b)

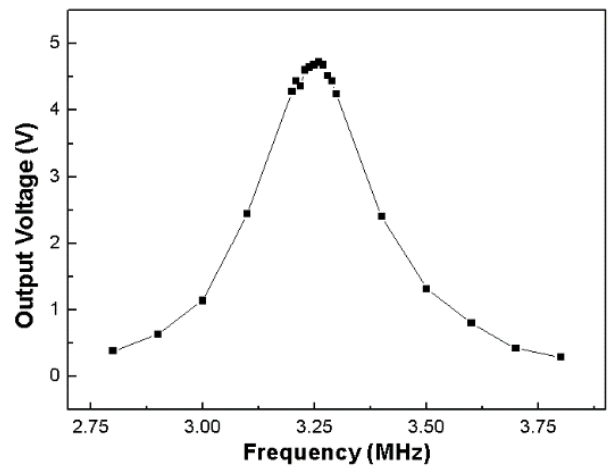

(d)

Figure 6. (a) Schematic diagram of magnetic resonance coupling coils, (b) a photograph of wireless power transfer set up, (c) modeling of currents on each coil over a frequency range from $2 \mathrm{MHz}$ to $4 \mathrm{MHz}$, and (d) output voltage at near resonance frequency.

A circuit model for the experimental setup with one receiving coil pair driving a single load was set up around each of the four loops. The source frequency based on the transfer function was determined with the circuit model of Cannon et al [6]. Figure 6(c) presents a theoretical graph of a circuit model for a set of coupling coefficients, clearly showing the circuit model with selected parameter values and adequate behavior of the resonant couple system with a single receiver. In WPT device design, capacitance values were chosen to yield identical resonant frequency with different sizes of source and receiver coils. In our study, resonance frequency was tuned at $3.26 \mathrm{MHz}$ with the output voltage of $4.8 \mathrm{~V}$. With an iterative study of the design parameters, $150 \mathrm{~mW}$ power output and $15.4 \%$ of transfer efficiency was obtained. Based on this promising outcome, a magnetic resonance coupling module including a feedback controlled source power supply was being developed.

\section{CONCLUSION}

Wirelessly powered micro-spectrometer and probe-pin devices were investigated for real time and implanted optical dopamine sensing in the body, which can alleviate surgical procedures for battery change out for long term operation. The implanted neural PPD, together with a neural sensing electronics interface and WPT enabling real-time measurement and control/feedback, was demonstrated with high sensitivity to micromole dopamine concentration. Wireless power transfer 
based on rectenna and magnetic resonance coupling presents promising outcomes to supply electrical power for system operation without having to change the battery. This performance level and device size are sufficient for potential implementation into clinical cases. Currently, the system integration and miniaturization of an optical neurotransmitter sensing PPD with a WPT system is underway for semi-autonomous management of neural functions through a routine of sensing, processing, and control.

\section{ACKNOWLEDGEMENT}

This work is supported by the Space Act Agreement (SAA) \#26160 and the NASA Grant (NNX12AH22A). Special thanks are expressed to Dr. Jongsung Kim of Gacheon University who provided fluorescence dopamine sensing probes.

\section{REFERENCES}

[1] Stocco, A.; Lebiere, C; Anderson, J.R., "Conditional routing of information to the cortex: A model of the basal ganglia's role in cognitive coordination." Psychological Review 117 (2), 541-74 (2010).

[2] Weyhenmeyer, J. A.; Gallman, E. A., "Rapid Review of Neuroscience.” Mosby Elsevier, ISBN-10: 0323022618, 102, (2007).

[3] Prescott, I. A., Dostrovsky, J. O., Moro, E., Hodaie, M., Lozano, A. M. and Hutchison, W. D., "Levodopa enhances synaptic plasticity in the substantia nigra pars reticulate of Parkinson's disease patients." Brain 132, 309-318 (2009).

[4] Moro, E, Lang, A. E., "Criteria for deep-brain stimulation in Parkinson's disease: review and analysis." Expert Review of Neurotherapeutics 6 (11), 1695-705, (Nov 2006).

[5] Brown, W. C., "Experiments involving a microwave beam to power and position a helicopter," IEEE Trans. Aerosp. Electron. Syst. AES-5, 692-702 (1969).

[6] Cannon, C, Hoburg, J. F., Stancil, D. D., and Goldstein, S. C., "Magnetic resonant coupling as a potential means for wireless power transfer to multiple small receivers," IEEE Trans. Power Electronics 24, 1819-1825 (2009).

[7] Tesla, N., "Apparatus for transmission of electrical energy," U.S. Patent No. 649,621 (1900).

[8] Hanlon, E. B., Manoharan, R., Koo, T. W., Shafer, K. E., Motz, J. T., Fitzmaurice, M., Kramer, J. R., Itzkan, I, Dasari, R. R. and Feld, M. S., "Prospects for in vivo Raman spectroscopy," Phys. Med. Biol. 45, R1-59 (2000).

[9] Chaganti, K, Salakhutdinov, I., Avrutsky, I and Auner, G. W., “A simple miniature optical spectrometer with a planar waveguide grating coupler in combination with a plano-convex lens," Opt. Express 14, 4064-4072, (2006).

[10] Montgomery, P. C., Montaner, D., Manzardo, O., Flury, M. and Herzig, H. P., "The metrology of a miniature FT spectrometer MOEMS device using white light scanning interference microscopy," Thin Solid Films 450, 79-83 (2004).

[11] Kitaura, N., Ogata, S. and Mori, Y., "Spectrometer employing a micro-Fresnel lens," Opt. Eng. 34, 584-588 (1995).

[12] Park, Y., Koch, L., Park, S. J., King, G. C., Song, K. D., and Choi, S. H., "Miniaturization of a Fresnel Spectrometer," Journal of Optics A: Pure and Applied Optics 10, (2008).

[13] Park, Y, Choi, S. H., "Miniaturization of optical spectroscopes into Fresnel microspectrometers," $J$. Nanophotonics 7, 077599-1, (2013).

[14] Kim, M. H., Song, K. D., Yoon, H., Park, Y, Choi, S. H., Lee, D. S., Shin, K. S., Hwang, H. I., Lee, U, “Probepin device for optical neurotransmitter sensing in the brain," SPIE Proc. 9434, 943409 (2015). 